\title{
Noether Theorem for Nonholonomic Systems with Time Delay
}

\author{
Shi-Xin Jin ${ }^{1}$ and Yi Zhang ${ }^{2}$ \\ ${ }^{1}$ College of Science, Nanjing University of Science and Technology, Nanjing, Jiangsu 210094, China \\ ${ }^{2}$ College of Civil Engineering, Suzhou University of Science and Technology, Suzhou, Jiangsu 215011, China \\ Correspondence should be addressed to Yi Zhang; zhy@mail.usts.edu.cn
}

Received 26 December 2014; Revised 16 March 2015; Accepted 16 March 2015

Academic Editor: Yuri Vladimirovich Mikhlin

Copyright (c) 2015 S.-X. Jin and Y. Zhang. This is an open access article distributed under the Creative Commons Attribution License, which permits unrestricted use, distribution, and reproduction in any medium, provided the original work is properly cited.

\begin{abstract}
The paper focuses on studying the Noether theorem for nonholonomic systems with time delay. Firstly, the differential equations of motion for nonholonomic systems with time delay are established, which is based on the Hamilton principle with time delay and the Lagrange multiplier rules. Secondly, based upon the generalized quasi-symmetric transformations for nonconservative systems with time delay, the Noether theorem for corresponding holonomic systems is given. Finally, we obtain the Noether theorem for the nonholonomic nonconservative systems with time delay. At the end of the paper, an example is given to illustrate the application of the results.
\end{abstract}

\section{Introduction}

Time delay is a lag phenomenon of time which is commonly found in nature and engineering practice. It is a more essential and realistic description of a mechanical system. Once we consider the influence of time delay, even a very simple problem, its dynamical behavior may become very complex [1$3]$. The variational problem with time delay was first studied and discussed by El'sgol'c [4]. In 1968, Hughes [5] discussed the variational and optimal control problems with time delay and obtained the analog of Euler-Lagrange equations with time delay. In addition, the variational problems with time delay were also discussed by Palm and Schmitendorf [6], Rosenblueth [7], Chan and Yung [8], Lee and Yung [9], and so on. A Bliss-type multiplier rule for constrained variational problems with time delay was discussed by Agrawal et al. [10].

The concept of symmetry plays an important role in both science and engineering. The importance of symmetry ranges from fundamental and theoretical aspects to concrete applications [11-21]. The symmetry is described by the invariance under an infinitesimal transformation. The symmetry of a mechanical system has great influence on its dynamical behavior. For example, the symmetry of time translation will lead to the conservation of energy of the system, the symmetry of space translation or the homogeneous of space will cause the conservation of momentum of the system, the symmetry of space rotation or the isotropy of space will result in the conservation of angular momentum of the system, and the conjugate symmetry of electric charge will lead to the conservation of electric quantity [22]. The influence on dynamical behavior for the symmetry is not limited to mechanical systems. For example, in crystallization kinetics of polymers, the higher the symmetry is and the better the regularity of the chain of molecular is, the easier the highly ordered regularly arranged lattice is formed [23]. Therefore, the study of the symmetries and the conserved quantities for mechanical systems with time delay is of great significance for in-depth understanding of the complex dynamics behavior and inherent physics nature. In 2012, the Noether symmetry theorems for variational and optimal control problems with time delay were first discussed by Frederico and Torres [24]. In 2013, the Noether symmetries for dynamics of nonconservative Lagrange system, nonconservative Hamilton system, and Birkhoffian system with time delay were studied by Zhang and his coworkers [25-27], and the relationship between the Noether symmetries and the conserved quantities was established. Up to now, more and more dynamical problems with time delay and its relevant mathematical theories are presented. However, the Noether theorem for nonholonomic systems with time delay has not been investigated yet in the literature. 
It is well known that a nonholonomic constraint is a differential constraint which is nonintegrable. The nonholonomic constraint is different from the holonomic constraint. The mathematical representation of nonholonomic constraint equations can correspond to a variety of realizations for constraint forces. Several models are allowed for the dynamics of nonholonomic systems such as the Chetaev model started from the d'Alembert-Lagrange principle [28, 29 ] and the Vacco model started from the Hamiltonian principle [30]. The differences between a nonholonomic system and a holonomic system are that the motion of a holonomic system can be described by the Lagrange equations, while the motion of a nonholonomic system is presented by more complex differential equations [28-32].

In this paper, we further extend the Noether theorem of nonconservative systems with time delay to nonholonomic nonconservative systems with time delay. The structure of this paper is organized as follows. In Section 2, we establish the differential equations of motion with time delay. In Section 3, we obtain the two formulae of variation for Hamilton action with time delay. In Section 4, the generalized Noether quasi-symmetric transformations are defined. In Section 5, the Noether theorem for corresponding holonomic system is given. In Section 6, we also obtain the Noether theorem for nonholonomic system with time delay. In Section 7 , an example is given to illustrate the application of the results.

\section{Differential Equations of Motion for Nonholonomic Systems with Time Delay}

Assume that the configuration of a mechanical systems is determined by $n$ generalized coordinates $q_{s}(s=1,2, \ldots, n)$. The motion of the system is subjected to $g$ bilateral ideal nonholonomic constraints of Chetaev type

$$
f_{\beta}\left(t, q_{s}, \dot{q}_{s}\right)=0 \quad(\beta=1,2, \ldots, g) .
$$

The restriction of constraints (1) exerted on the virtual displacements is

$$
\frac{\partial f_{\beta}}{\partial \dot{q}_{s}} \delta q_{s}=0 .
$$

The Hamilton principle for nonconservative systems is [14]

$$
\int_{t_{1}}^{t_{2}}\left(\delta L+Q_{s}^{\prime \prime} \delta q_{s}\right) d t=0,
$$

where $L$ is the Lagrangian and $Q_{s}^{\prime \prime}$ are the generalized nonpotential forces. Considering that the time delay exists, the Lagrangian is $L=L\left(t, q_{s}, \dot{q}_{s}, q_{s}(t-\tau), \dot{q}_{s}(t-\tau)\right)=$ $L\left(t, q_{s}, \dot{q}_{s}, q_{s \tau}, \dot{q}_{s \tau}\right)$ and the generalized nonpotential forces are $Q_{s}^{\prime \prime}=Q_{s}^{\prime \prime}\left(t, q_{s}, \dot{q}_{s}, q_{s \tau}, \dot{q}_{s \tau}\right)$. The boundary conditions

$$
\begin{array}{ll}
q_{s}(t)=\Omega_{s}(t), & t \in\left[t_{1}-\tau, t_{1}\right], \\
q_{s}(t)=q_{s}\left(t_{2}\right), & t=t_{2}
\end{array}
$$

are satisfied, where $\tau$ is a given positive real number such that $\tau<t_{2}-t_{1}, \Omega_{s}(t)$ are given piecewise smooth functions in $\left[t_{1}-\tau, t_{1}\right]$, and $q_{s}\left(t_{2}\right)$ are certain values. From formulae (2) and (3), we have

$$
\begin{gathered}
\int_{t_{1}}^{t_{2}}\left[\frac{\partial L}{\partial q_{s}}(t) \delta q_{s}+\frac{\partial L}{\partial \dot{q}_{s}}(t) \delta \dot{q}_{s}+\frac{\partial L}{\partial q_{s \tau}}(t) \delta q_{s \tau}+\frac{\partial L}{\partial \dot{q}_{s \tau}}(t) \delta \dot{q}_{s \tau}\right. \\
\left.+Q_{s}^{\prime \prime}(t) \delta q_{s}+\lambda_{\beta} \frac{\partial f_{\beta}}{\partial \dot{q}_{s}}(t) \delta q_{s}\right] d t=0,
\end{gathered}
$$

where $\lambda_{\beta}$ is the Lagrange multiplier. By the change of variable $t=\theta+\tau$ in the third and fourth terms in formula (6) and considering condition (4), we have

$$
\begin{aligned}
\int_{t_{1}}^{t_{2}} & \left(\frac{\partial L}{\partial q_{s \tau}}(t) \delta q_{s \tau}+\frac{\partial L}{\partial \dot{q}_{s \tau}}(t) \delta \dot{q}_{s \tau}\right) d t \\
\quad= & \int_{t_{1}}^{t_{2}-\tau}\left(\frac{\partial L}{\partial q_{s \tau}}(\theta+\tau) \delta q_{s}+\frac{\partial L}{\partial \dot{q}_{s \tau}}(\theta+\tau) \delta \dot{q}_{s}\right) d \theta
\end{aligned}
$$

Substituting (7) into (6), we have

$$
\begin{gathered}
\int_{t_{1}}^{t_{2}-\tau}\left[\frac{\partial L}{\partial q_{s}}(t) \delta q_{s}+\frac{\partial L}{\partial \dot{q}_{s}}(t) \delta \dot{q}_{s}+\frac{\partial L}{\partial q_{s \tau}}(t+\tau) \delta q_{s}\right. \\
\left.+\frac{\partial L}{\partial \dot{q}_{s \tau}}(t+\tau) \delta \dot{q}_{s}+Q_{s}^{\prime \prime}(t) \delta q_{s}+\lambda_{\beta} \frac{\partial f_{\beta}}{\partial \dot{q}_{s}}(t) \delta q_{s}\right] d t \\
+\int_{t_{2}-\tau}^{t_{2}}\left[\frac{\partial L}{\partial q_{s}}(t) \delta q_{s}+\frac{\partial L}{\partial \dot{q}_{s}}(t) \delta \dot{q}_{s}+Q_{s}^{\prime \prime}(t) \delta q_{s}\right. \\
\left.+\lambda_{\beta} \frac{\partial f_{\beta}}{\partial \dot{q}_{s}}(t) \delta q_{s}\right] d t=0 .
\end{gathered}
$$

Integrating by parts and taking use of boundary conditions (4) and (5), we have

$$
\begin{gathered}
\int_{t_{1}}^{t_{2}-\tau}\left(\frac{\partial L}{\partial q_{s}}(t)+\frac{\partial L}{\partial q_{s \tau}}(t+\tau)\right. \\
\left.+Q_{s}^{\prime \prime}(t)+\lambda_{\beta} \frac{\partial f_{\beta}}{\partial \dot{q}_{s}}(t)\right) \delta q_{s} d t \\
=-\left[\delta q _ { s } \int _ { t } ^ { t _ { 2 } - \tau } \left(\frac{\partial L}{\partial q_{s}}(\theta)+\frac{\partial L}{\partial q_{s \tau}}(\theta+\tau)+Q_{s}^{\prime \prime}(\theta)\right.\right. \\
+\int_{t_{1}}^{t_{2}-\tau} \delta \dot{q}_{s}\left[\int_{t}^{t_{2}-\tau}\left(\frac{\partial L}{\partial q_{s}}(\theta)+\frac{\partial L}{\partial q_{s \tau}}(\theta+\tau)+Q_{s}^{\prime \prime}(\theta) d \theta\right]_{t_{1}}^{t_{2}-\tau}\right. \\
+\dot{q}_{s} \\
+\int_{t_{1}}^{t_{2}-\tau} \delta \dot{q}_{s}\left[\int_{t}^{t_{2}-\tau}\left(\frac{\partial L}{\partial q_{s}}(\theta)+\frac{\partial L}{\partial \dot{q}_{s \tau}}(\theta)\right) d \theta\right] d t \\
\left.\left.+\lambda_{\beta} \frac{\partial f_{\beta}}{\partial \dot{q}_{s}}(\theta)\right) d \theta\right] d t
\end{gathered}
$$




$$
\begin{gathered}
\int_{t_{2}-\tau}^{t_{2}}\left(\frac{\partial L}{\partial q_{s}}(t)+Q_{s}^{\prime \prime}(t)+\lambda_{\beta} \frac{\partial f_{\beta}}{\partial q_{s}}(t)\right) \delta q_{s} d t \\
=\left[\delta q _ { s } \int _ { t _ { 2 } - \tau } ^ { t } \left(\frac{\partial L}{\partial q_{s}}(\theta)+Q_{s}^{\prime \prime}(\theta)\right.\right. \\
\left.\left.+\lambda_{\beta} \frac{\partial f_{\beta}}{\partial \dot{q}_{s}}(\theta)\right) d \theta\right]_{t_{2}-\tau}^{t_{2}} \\
-\int_{t_{2}-\tau}^{t_{2}} \delta \dot{q}_{s}\left[\int _ { t _ { 2 } - \tau } ^ { t } \left(\frac{\partial L}{\partial q_{s}}(\theta)+Q_{s}^{\prime \prime}(\theta)\right.\right. \\
\left.\left.+\lambda_{\beta} \frac{\partial f_{\beta}}{\partial \dot{q}_{s}}(\theta)\right) d \theta\right] d t \\
=-\int_{t_{2}-\tau}^{t_{2}} \delta \dot{q}_{s}\left[\int _ { t _ { 2 } - \tau } ^ { t } \left(\frac{\partial L}{\partial q_{s}}(\theta)+Q_{s}^{\prime \prime}(\theta)\right.\right. \\
\left.\left.+\lambda_{\beta} \frac{\partial f_{\beta}}{\partial \dot{q}_{s}}(\theta)\right) d \theta\right] d t .
\end{gathered}
$$

Substituting (9) into (8), we have

$$
\begin{gathered}
\int_{t_{1}}^{t_{2}-\tau} \delta \dot{q}_{s}\left[\int _ { t } ^ { t _ { 2 } - \tau } \left(\frac{\partial L}{\partial q_{s}}(\theta)+\frac{\partial L}{\partial q_{s \tau}}(\theta+\tau)+Q_{s}^{\prime \prime}(\theta)\right.\right. \\
\left.+\lambda_{\beta} \frac{\partial f_{\beta}}{\partial \dot{q}_{s}}(\theta)\right) d \theta+\frac{\partial L}{\partial \dot{q}_{s \tau}}(t+\tau) \\
\left.+\frac{\partial L}{\partial \dot{q}_{s}}(t)\right] d t \\
+\int_{t_{2}-\tau}^{t_{2}}\left[-\int_{t_{2}-\tau}^{t}\left(\frac{\partial L}{\partial q_{s}}(\theta)+Q_{s}^{\prime \prime}(\theta)+\lambda_{\beta} \frac{\partial f_{\beta}}{\partial \dot{q}_{s}}(\theta)\right) d \theta\right. \\
\left.+\frac{\partial L}{\partial \dot{q}_{s}}\right] \delta \dot{q}_{s} d t=0 .
\end{gathered}
$$

Considering the arbitrariness of the integral interval and taking use of the Lagrange multipliers rules, we have

$$
\begin{array}{r}
\int_{t}^{t_{2}-\tau}\left(\frac{\partial L}{\partial q_{s}}(\theta)+\frac{\partial L}{\partial q_{s \tau}}(\theta+\tau)+Q_{s}^{\prime \prime}(\theta)+\lambda_{\beta} \frac{\partial f_{\beta}}{\partial \dot{q}_{s}}(\theta)\right) d \theta \\
+\frac{\partial L}{\partial \dot{q}_{s}}(t)+\frac{\partial L}{\partial \dot{q}_{s \tau}}(t+\tau)=0, \quad t \in\left[t_{1}, t_{2}-\tau\right] \\
-\int_{t_{2}-\tau}^{t}\left(\frac{\partial L}{\partial q_{s}}(\theta)+Q_{s}^{\prime \prime}(\theta)+\lambda_{\beta} \frac{\partial f_{\beta}}{\partial \dot{q}_{s}}(\theta)\right) d \theta+\frac{\partial L}{\partial \dot{q}_{s}}(t)=0, \\
t \in\left(t_{2}-\tau, t_{2}\right] .
\end{array}
$$

Taking derivative of (11) with respect to $t$, we have

$$
\begin{gathered}
\frac{d}{d t} \frac{\partial L}{\partial \dot{q}_{s}}(t)+\frac{d}{d t} \frac{\partial L}{\partial \dot{q}_{s \tau}}(t+\tau)-\frac{\partial L}{\partial q_{s}}(t)-\frac{\partial L}{\partial q_{s \tau}}(t+\tau) \\
=Q_{s}^{\prime \prime}(t)+\lambda \lambda_{\beta} \frac{\partial f_{\beta}}{\partial \dot{q}_{s}}(t), \quad t \in\left[t_{1}, t_{2}-\tau\right] \\
\frac{d}{d t} \frac{\partial L}{\partial \dot{q}_{s}}(t)-\frac{\partial L}{\partial q_{s}}(t)=Q_{s}^{\prime \prime}(t)+\lambda_{\beta} \frac{\partial f_{\beta}}{\partial \dot{q}_{s}}(t), \\
t \in\left(t_{2}-\tau, t_{2}\right], \quad(s=1,2, \ldots, n) .
\end{gathered}
$$

Equations in (12) are called the differential equations of motion for nonholonomic systems with time delay. Before integrating the differential equations of motion, by using (1) and (12) one can find $\lambda_{\beta}$ as the functions of $t, q_{s}, \dot{q}_{s}, q_{s \tau}$, and $\dot{q}_{s \tau}$. Therefore, (12) can be written in the form

$$
\begin{gathered}
\frac{d}{d t} \frac{\partial L}{\partial \dot{q}_{s}}(t)+\frac{d}{d t} \frac{\partial L}{\partial \dot{q}_{s \tau}}(t+\tau)-\frac{\partial L}{\partial q_{s}}(t)-\frac{\partial L}{\partial q_{s \tau}}(t+\tau) \\
=Q^{\prime \prime}(t)_{s}+\Lambda_{s}(t), \quad t \in\left[t_{1}, t_{2}-\tau\right], \\
\frac{d}{d t} \frac{\partial L}{\partial \dot{q}_{s}}(t)-\frac{\partial L}{\partial q_{s}}(t)=Q_{s}^{\prime \prime}(t)+\Lambda_{s}(t), \\
t \in\left(t_{2}-\tau, t_{2}\right], \quad(s=1,2, \ldots, n),
\end{gathered}
$$

where

$$
\Lambda_{s}=\lambda_{\beta} \frac{\partial f_{\beta}}{\partial \dot{q}_{s}}, \quad(s=1,2, \ldots, n) .
$$

Equations in (13) are called the differential equations for corresponding holonomic systems with time delay. If the initial conditions satisfy the equations of nonholonomic constraints (1), then the solution of the corresponding holonomic systems (13) will give the motion of the nonholonomic systems. If $\tau=0$, then equations in (12) are reduced to the differential equations of motion for the standard nonholonomic systems.

\section{Variation of Hamilton Actions with Time Delay}

The Hamilton action with time delay is

$$
\begin{aligned}
S(\gamma) & =\int_{t_{1}}^{t_{2}} L\left(t, q_{s}(t), \dot{q}_{s}(t), q_{s}(t-\tau), \dot{q}_{s}(t-\tau)\right) d t \\
& \triangleq \int_{t_{1}}^{t_{2}} L\left(t, q_{s}, \dot{q}_{s}, q_{s \tau}, \dot{q}_{s \tau}\right) d t,
\end{aligned}
$$

where $\gamma$ is a curve. Introduce the infinitesimal transformations of $r$ parameter finite transformations of group

$$
\bar{t}=t+\Delta t, \quad \bar{q}_{s}(\bar{t})=q_{s}(t)+\Delta q_{s}
$$

or their expansion formulae

$$
\begin{array}{r}
\bar{t}=t+\varepsilon_{\sigma} \xi_{0}^{\sigma}\left(t, q_{k}, \dot{q}_{k}\right), \quad \bar{q}_{s}(\bar{t})=q_{s}(t)+\varepsilon_{\sigma} \xi_{s}^{\sigma}\left(t, q_{k}, \dot{q}_{k}\right) \\
(s, k=1,2, \ldots, n),
\end{array}
$$


where $\varepsilon_{\sigma}(\sigma=1,2, \ldots, r)$ are the infinitesimal parameters and $\xi_{0}^{\sigma}, \xi_{s}^{\sigma}$ are the generators or generating functions for the infinitesimal transformations. Under the infinitesimal transformations (16), the Hamilton action (15) can be expressed as

$$
S(\gamma)=\int_{\bar{t}_{1}}^{\bar{t}_{2}} L\left(\bar{t}, \bar{q}_{s}(\bar{t}), \dot{\bar{q}}_{s}(\bar{t}), \bar{q}_{s}(\bar{t}-\tau), \dot{\bar{q}}_{s}(\bar{t}-\tau)\right) d \bar{t},
$$

where $\bar{\gamma}$ is a neighbor curve of $\gamma$. Then, the main linear part relative to $\varepsilon$ in the difference $S(\bar{\gamma})-S(\gamma)$ is

$$
\begin{gathered}
\Delta S=\int_{t_{1}}^{t_{2}}\left[\frac{\partial L}{\partial t} \Delta t+\frac{\partial L}{\partial q_{s}} \Delta q_{s}+\frac{\partial L}{\partial \dot{q}_{s}} \Delta \dot{q}_{s}+\frac{\partial L}{\partial q_{s \tau}} \Delta q_{s \tau}\right. \\
\left.+\frac{\partial L}{\partial \dot{q}_{s \tau}} \Delta \dot{q}_{s \tau}+L \frac{d}{d t}(\Delta t)\right] d t .
\end{gathered}
$$

By making a linear change of variable $t=\theta+\tau$ in the fourth and fifth terms under formula (19) and considering the boundary conditions (4), we have

$$
\begin{aligned}
& \int_{t_{1}}^{t_{2}}\left(\frac{\partial L}{\partial q_{s \tau}}(t) \Delta q_{s \tau}+\frac{\partial L}{\partial \dot{q}_{s \tau}}(t) \Delta \dot{q}_{s \tau}\right) d t \\
& \quad=\int_{t_{1}}^{t_{2}-\tau}\left(\frac{\partial L}{\partial q_{s \tau}}(\theta+\tau) \Delta q_{s}(\theta)+\frac{\partial L}{\partial \dot{q}_{s \tau}}(\theta+\tau) \Delta \dot{q}_{s}(\theta)\right) d \theta .
\end{aligned}
$$

Substituting formula (20) into (19), we have

$$
\begin{aligned}
\Delta S=\int_{t_{1}}^{t_{2}-\tau}[ & \frac{\partial L}{\partial t}(t) \Delta t+\frac{\partial L}{\partial q_{s}}(t) \Delta q_{s}+\frac{\partial L}{\partial \dot{q}_{s}}(t) \Delta \dot{q}_{s} \\
& +\frac{\partial L}{\partial q_{s \tau}}(t+\tau) \Delta q_{s}+\frac{\partial L}{\partial \dot{q}_{s \tau}}(t+\tau) \Delta \dot{q}_{s} \\
& \left.+L \frac{d}{d t}(\Delta t)\right] d t \\
+\int_{t_{2}-\tau}^{t_{2}} & {\left[\frac{\partial L}{\partial t}(t) \Delta t+\frac{\partial L}{\partial q_{s}}(t) \Delta q_{s}+\frac{\partial L}{\partial \dot{q}_{s}}(t) \Delta \dot{q}_{s}\right.} \\
& \left.+L \frac{d}{d t}(\Delta t)\right] d t .
\end{aligned}
$$

Noticing that

$$
\Delta \dot{q}_{s}=\frac{d}{d t} \Delta q_{s}-\dot{q}_{s} \frac{d}{d t} \Delta t, \quad \delta q_{s}=\Delta q_{s}-\dot{q}_{s} \Delta t
$$

and transformations (16), we can express formula (21) as

$$
\begin{aligned}
\Delta S=\int_{t_{1}}^{t_{2}-\tau} \varepsilon_{\sigma}\left\{\frac{d}{d t}\left[L \xi_{0}^{\sigma}+\left(\frac{\partial L}{\partial \dot{q}_{s}}(t)+\frac{\partial L}{\partial \dot{q}_{s \tau}}(t+\tau)\right) \bar{\xi}_{s}^{\sigma}\right]\right. \\
+\left[\frac{\partial L}{\partial q_{s}}(t)+\frac{\partial L}{\partial q_{s \tau}}(t+\tau)-\frac{d}{d t}\right. \\
\left.\left.\cdot\left(\frac{\partial L}{\partial \dot{q}_{s}}(t)+\frac{\partial L}{\partial \dot{q}_{s \tau}}(t+\tau)\right)\right] \bar{\xi}_{s}^{\sigma}\right\} d t \\
+\int_{t_{2}-\tau}^{t_{2}} \varepsilon_{\sigma}\left\{\frac{d}{d t}\left[L \xi_{0}^{\sigma}+\frac{\partial L}{\partial \dot{q}_{s}}(t) \bar{\xi}_{s}^{\sigma}\right]\right. \\
\left.+\left(\frac{\partial L}{\partial q_{s}}(t)-\frac{d}{d t} \frac{\partial L}{\partial \dot{q}_{s}}(t)\right) \bar{\xi}_{s}^{\sigma}\right\} d t
\end{aligned}
$$

where

$$
\bar{\xi}_{s}^{\sigma}=\xi_{s}^{\sigma}-\dot{q}_{s} \xi_{0}^{\sigma} .
$$

Formulae (21) and (23) are basic formulae for the variation of Hamilton action with time delay.

\section{Generalized Noether Quasi-Symmetric Transformations with Time Delay}

The Noether symmetry is an invariance of Hamilton action under the group of infinitesimal transformations. Therefore, we have the following.

Definition 1. If the Hamilton action with time delay is invariant under the group of infinitesimal transformations, that is, for each of the infinitesimal transformations the formula

$$
\Delta S=0
$$

holds, then the infinitesimal transformations are called the Noether symmetric transformations of dynamics systems with time delay.

Definition 2. If the Hamilton action with time delay is quasiinvariant under the group of infinitesimal transformations, that is, for each of the infinitesimal transformations the formula

$$
\Delta S=-\int_{t_{1}}^{t_{2}} \frac{d}{d t}(\Delta G) d t
$$

holds, where $G=G\left(t, q_{s}(t), \dot{q}_{s}(t), q_{s}(t-\tau), \dot{q}_{s}(t-\tau)\right)$ is a gauge function, then the infinitesimal transformations are called the Noether quasi-symmetric transformations of dynamics systems with time delay.

Definition 3. If the Hamilton action with time delay is generalized quasi-invariant under the group of infinitesimal transformations, that is, for each of the infinitesimal transformations the formula

$$
\Delta S=-\int_{t_{1}}^{t_{2}}\left(\frac{d}{d t}(\Delta G)+Q_{s}^{\prime \prime} \delta q_{s}\right) d t
$$


holds, then the infinitesimal transformations are called the generalized Noether quasi-symmetric transformations of dynamics systems with time delay.

From Definition 3 and the formula (21), we obtain the following.

Theorem 4. If the infinitesimal transformations (16) satisfy the conditions

$$
\begin{aligned}
& \frac{\partial L}{\partial t}(t) \Delta t+\left(\frac{\partial L}{\partial q_{s}}(t)+\frac{\partial L}{\partial q_{s \tau}}(t+\tau)\right) \Delta q_{s} \\
& +\left(\frac{\partial L}{\partial \dot{q}_{s}}(t)+\frac{\partial L}{\partial \dot{q}_{s \tau}}(t+\tau)\right) \Delta \dot{q}_{s}+Q_{s}^{\prime \prime}(t)\left(\Delta q_{s}-\dot{q}_{s} \Delta t\right) \\
& +L \frac{d}{d t}(\Delta t)=-\frac{d}{d t}(\Delta G)
\end{aligned}
$$

for $t_{1} \leq t \leq t_{2}-\tau$, and

$$
\begin{aligned}
& \frac{\partial L}{\partial t}(t) \Delta t+\frac{\partial L}{\partial q_{s}}(t) \Delta q_{s}+\frac{\partial L}{\partial \dot{q}_{s}}(t) \Delta \dot{q}_{s}+Q_{s}^{\prime \prime}(t)\left(\Delta q_{s}-\dot{q}_{s} \Delta t\right) \\
& \quad+L \frac{d}{d t}(\Delta t)=-\frac{d}{d t}(\Delta G)
\end{aligned}
$$

for $t_{2}-\tau<t \leq t_{2}$, then the infinitesimal transformations are the generalized Noether quasi-symmetric transformations in the sense of Definition 3.

Theorem 4 can also be expressed as the following theorem.

Theorem 5. If the infinitesimal transformations (17) satisfy the conditions

$$
\begin{aligned}
& \frac{\partial L}{\partial t}(t) \xi_{0}^{\sigma}+\left(\frac{\partial L}{\partial q_{s}}(t)+\frac{\partial L}{\partial q_{s \tau}}(t+\tau)\right) \xi_{s}^{\sigma} \\
& +\left(\frac{\partial L}{\partial \dot{q}_{s}}(t)+\frac{\partial L}{\partial \dot{q}_{s \tau}}(t+\tau)\right)\left(\dot{\xi}_{s}^{\sigma}-\dot{q}_{s} \dot{\xi}_{0}^{\sigma}\right) \\
& +Q_{s}^{\prime \prime}(t) \bar{\xi}_{s}^{\sigma}+L \dot{\xi}_{0}^{\sigma}=-\dot{G}^{\sigma}, \quad(\sigma=1,2, \ldots, r),
\end{aligned}
$$

for $t_{1} \leq t \leq t_{2}-\tau$, and

$$
\begin{aligned}
& \frac{\partial L}{\partial t}(t) \xi_{0}^{\sigma}+\frac{\partial L}{\partial q_{s}}(t) \xi_{s}^{\sigma}+\frac{\partial L}{\partial \dot{q}_{s}}(t)\left(\dot{\xi}_{s}^{\sigma}-\dot{q}_{s} \dot{\xi}_{0}^{\sigma}\right) \\
& \quad+Q_{s}^{\prime \prime}(t) \bar{\xi}_{s}^{\sigma}+L \dot{\xi}_{0}^{\sigma}=-\dot{G}^{\sigma}, \quad(\sigma=1,2, \ldots, r)
\end{aligned}
$$

for $t_{2}-\tau<t \leq t_{2}$, where $\Delta G=\varepsilon_{\sigma} G^{\sigma}$, then the infinitesimal transformations are the generalized Noether quasi-symmetric transformations in the sense of Definition 3.

When $r=1$, formulae (30) and (31) are called the Noether identity of dynamics systems with time delay. By using Theorem 4 or Theorem 5 , we can verify the generalized Noether quasi-symmetry with time delay.

\section{Noether Theorem of Corresponding Holonomic Systems with Time Delay}

For the corresponding holonomic systems (13) with time delay, if we can find a generalized Noether quasi-symmetric transformation, then we can get a corresponding conserved quantity. So we have the following.

Theorem 6. For the corresponding holonomic systems (13) with time delay, if the infinitesimal transformations (17) are the generalized quasi-symmetric transformations in the sense of Definition 3, that is, the transformations satisfy the conditions

$$
\begin{aligned}
& \frac{\partial L}{\partial t}(t) \xi_{0}^{\sigma}+\left[\frac{\partial L}{\partial q_{s}}(t)+\frac{\partial L}{\partial q_{s \tau}}(t+\tau)\right] \xi_{s}^{\sigma} \\
& +\left[L-\left(\frac{\partial L}{\partial \dot{q}_{s}}(t)+\frac{\partial L}{\partial \dot{q}_{s \tau}}(t+\tau)\right) \dot{q}_{s}\right] \dot{\xi}_{0}^{\sigma} \\
& +\left[\frac{\partial L}{\partial \dot{q}_{s}}(t)+\frac{\partial L}{\partial \dot{q}_{s \tau}}(t+\tau)\right] \dot{\xi}_{s}^{\sigma} \\
& +\left(Q_{s}^{\prime \prime}(t)+\Lambda_{s}(t)\right) \bar{\xi}_{s}^{\sigma}=-\dot{G}^{\sigma}
\end{aligned}
$$

for $t_{1} \leq t \leq t_{2}-\tau$ and

$$
\begin{gathered}
\frac{\partial L}{\partial t}(t) \xi_{0}^{\sigma}+\frac{\partial L}{\partial q_{s}}(t) \xi_{s}^{\sigma}+\frac{\partial L}{\partial \dot{q}_{s}}(t) \dot{\xi}_{s}^{\sigma}+\left(L-\frac{\partial L}{\partial \dot{q}_{s}}(t) \dot{q}_{s}\right) \dot{\xi}_{0}^{\sigma} \\
+\left(Q_{s}^{\prime \prime}(t)+\Lambda_{s}(t)\right) \bar{\xi}_{s}^{\sigma}=-\dot{G}^{\sigma}, \quad(\sigma=1,2, \ldots, r)
\end{gathered}
$$

for $t_{2}-\tau<t \leq t_{2}$, then there exists a system of $r$ linear independent conserved quantities

$$
I^{\sigma}=L \xi_{0}^{\sigma}+\left(\frac{\partial L}{\partial \dot{q}_{s}}(t)+\frac{\partial L}{\partial \dot{q}_{s \tau}}(t+\tau)\right) \bar{\xi}_{s}^{\sigma}+G^{\sigma}=\text { const. }
$$

for $t_{1} \leq t \leq t_{2}-\tau$, and

$$
I^{\sigma}=L \xi_{0}^{\sigma}+\frac{\partial L}{\partial \dot{q}_{s}}(t) \bar{\xi}_{s}^{\sigma}+G^{\sigma}=\text { const. }, \quad(\sigma=1,2, \ldots, r)
$$

for $t_{2}-\tau<t \leq t_{2}$

Proof. From formulae (32) and (33), we have

$$
\begin{aligned}
\frac{d}{d t}\left[L \xi_{0}^{\sigma}\right. & \left.+\left(\frac{\partial L}{\partial \dot{q}_{s}}(t)+\frac{\partial L}{\partial \dot{q}_{s \tau}}(t+\tau)\right) \bar{\xi}_{s}^{\sigma}+G^{\sigma}\right] \\
+\bar{\xi}_{s}^{\sigma} & {\left[\frac{\partial L}{\partial q_{s}}(t)+\frac{\partial L}{\partial q_{s \tau}}(t+\tau)-\frac{d}{d t} \frac{\partial L}{\partial \dot{q}_{s}}(t)\right.} \\
& \left.-\frac{d}{d t} \frac{\partial L}{\partial \dot{q}_{s \tau}}(t+\tau)+Q_{s}^{\prime \prime}(t)+\Lambda_{s}(t)\right]=0
\end{aligned}
$$


for $t_{1} \leq t \leq t_{2}-\tau$, and

$$
\begin{aligned}
& \frac{d}{d t}\left[L \xi_{0}^{\sigma}+\frac{\partial L}{\partial \dot{q}_{s}}(t) \bar{\xi}_{s}^{\sigma}+G^{\sigma}\right] \\
&+\left[\frac{\partial L}{\partial q_{s}}(t)-\frac{d}{d t} \frac{\partial L}{\partial \dot{q}_{s}}(t)+Q_{s}^{\prime \prime}(t)+\Lambda_{s}(t)\right]=0, \\
&(\sigma=1,2, \ldots, r)
\end{aligned}
$$

for $t_{2}-\tau<t \leq t_{2}$. Substituting (13) into formulae (36) and (37), we obtain

$$
\frac{d}{d t}\left[L \xi_{0}^{\sigma}+\left(\frac{\partial L}{\partial \dot{q}_{s}}(t)+\frac{\partial L}{\partial \dot{q}_{s \tau}}(t+\tau)\right) \bar{\xi}_{s}^{\sigma}+G^{\sigma}\right]=0
$$

for $t_{1} \leq t \leq t_{2}-\tau$ and

$$
\frac{d}{d t}\left(L \xi_{0}^{\sigma}+\frac{\partial L}{\partial \dot{q}_{s}}(t) \bar{\xi}_{s}^{\sigma}+G^{\sigma}\right)=0, \quad(\sigma=1,2, \ldots, r)
$$

for $t_{2}-\tau<t \leq t_{2}$. Integrating formulae (38) and (39), we get the results.

Theorem 6 can be called the Noether theorem for corresponding holonomic systems (13).

\section{Noether Theorem of Nonholonomic Systems with Time Delay}

Because

$$
\delta q_{s}=\Delta q_{s}-\dot{q}_{s} \Delta t=\varepsilon_{\sigma} \bar{\xi}_{s}^{\sigma}
$$

by considering the independent of $\delta \dot{q}_{s}$, formula (2) can be expressed as

$$
\frac{\partial f_{\beta}}{\partial \dot{q}_{s}} \bar{\xi}_{s}^{\sigma}=0, \quad(\beta=1,2, \ldots, g ; \sigma=1,2, \ldots, r) .
$$

This is the restriction of the nonholonomic constraints on the generating functions of the infinitesimal transformations, and formula (41) can be called the Appell-Chetaev condition.

Theorem 7. For the given nonholonomic systems (1) and (12) with time delay, if the infinitesimal transformations (16) are the generalized Noether quasi-symmetrical transformations and satisfy the Appell-Chetaev condition (41), then there exists a systems of $r$ linear independent conserved quantities (34) and (35).
Proof. From Definition 3 and formula (23), we obtain

$$
\begin{gathered}
\int_{t_{1}}^{t_{2}-\tau} \varepsilon_{\sigma}\left\{\frac{d}{d t}\left[L \xi_{0}^{\sigma}+\left(\frac{\partial L}{\partial \dot{q}_{s}}(t)+\frac{\partial L}{\partial \dot{q}_{s \tau}}(t+\tau)\right) \bar{\xi}_{s}^{\sigma}+G^{\sigma}\right]\right. \\
+\bar{\xi}_{s}^{\sigma}\left[\frac{\partial L}{\partial q_{s}}(t)+\frac{\partial L}{\partial q_{s \tau}}(t+\tau)-\frac{d}{d t} \frac{\partial L}{\partial \dot{q}_{s}}(t)\right. \\
\left.\left.\quad-\frac{d}{d t} \frac{\partial L}{\partial \dot{q}_{s \tau}}(t+\tau)+Q_{s}^{\prime \prime}(t)\right]\right\} d t \\
+\int_{t_{2}-\tau}^{t_{2}} \varepsilon_{\sigma}\left[\frac{d}{d t}\left(L \xi_{0}^{\sigma}+\frac{\partial L}{\partial \dot{q}_{s}}(t) \bar{\xi}_{s}^{\sigma}+G^{\sigma}\right)\right. \\
\left.+\bar{\xi}_{s}^{\sigma}\left(\frac{\partial L}{\partial q_{s}}(t)-\frac{d}{d t} \frac{\partial L}{\partial \dot{q}_{s}}(t)+Q_{s}^{\prime \prime}(t)\right)\right] d t=0 .
\end{gathered}
$$

From the Appell-Chetaev condition (41), we have

$$
\int_{t_{1}}^{t_{2}} \varepsilon_{\sigma} \lambda_{\beta} \frac{\partial f_{\beta}}{\partial \dot{q}_{s}} \bar{\xi}_{s}^{\sigma} d t=0
$$

Adding (43) to (42), we obtain

$$
\begin{gathered}
\int_{t_{1}}^{t_{2}-\tau} \varepsilon_{\sigma}\left\{\frac{d}{d t}\left[L \xi_{0}^{\sigma}+\left(\frac{\partial L}{\partial \dot{q}_{s}}(t)+\frac{\partial L}{\partial \dot{q}_{s \tau}}(t+\tau)\right) \bar{\xi}_{s}^{\sigma}+G^{\sigma}\right]\right. \\
+\bar{\xi}_{s}^{\sigma}\left[\frac{\partial L}{\partial q_{s}}(t)+\frac{\partial L}{\partial q_{s \tau}}(t+\tau)-\frac{d}{d t} \frac{\partial L}{\partial \dot{q}_{s}}(t)\right. \\
\left.\left.-\frac{d}{d t} \frac{\partial L}{\partial \dot{q}_{s \tau}}(t+\tau)+Q_{s}^{\prime \prime}(t)+\lambda_{\beta} \frac{\partial f_{\beta}}{\partial \dot{q}_{s}}\right]\right\} d t \\
+\int_{t_{2}-\tau}^{t_{2}} \varepsilon_{\sigma}\left\{\frac{d}{d t}\left[L \xi_{0}^{\sigma}+\frac{\partial L}{\partial \dot{q}_{s}}(t) \bar{\xi}_{s}^{\sigma}+G^{\sigma}\right]\right. \\
+\bar{\xi}_{s}^{\sigma}\left(\frac{\partial L}{\partial q_{s}}(t)-\frac{d}{d t} \frac{\partial L}{\partial \dot{q}_{s}}(t)\right. \\
\left.\left.+Q_{s}^{\prime \prime}(t)+\lambda_{\beta} \frac{\partial f_{\beta}}{\partial \dot{q}_{s}}\right)\right\} d t=0 .
\end{gathered}
$$

Substituting (12) into formula (44) and considering the independence of $\varepsilon_{\sigma}$ and the arbitrariness of integration interval, we obtain

$$
\frac{d}{d t}\left[L \xi_{0}^{\sigma}+\left(\frac{\partial L}{\partial \dot{q}_{s}}(t)+\frac{\partial L}{\partial \dot{q}_{s \tau}}(t+\tau)\right) \bar{\xi}_{s}^{\sigma}+G^{\sigma}\right]=0
$$

for $t_{1} \leq t \leq t_{2}-\tau$ and

$$
\frac{d}{d t}\left(L \xi_{0}^{\sigma}+\frac{\partial L}{\partial \dot{q}_{s}} \bar{\xi}_{s}^{\sigma}+G^{\sigma}\right)=0, \quad(\sigma=1,2, \ldots, r)
$$

for $t_{2}-\tau<t \leq t_{2}$. Integrating formulae (45) and (46), we get the results.

Theorem 7 is called the Noether theorem for nonholonomic systems (1) and (12) with time delay. According to 
Theorem 7, if we can find a generalized Noether quasisymmetric transformation for nonholonomic systems with time delay, then we can get a conserved quantity of the systems.

\section{Illustrative Example}

Example. Considering the dynamic system with time delay, the Lagrangian is

$$
L=\frac{1}{2}\left[\dot{q}_{1}^{2}(t)+\dot{q}_{1}^{2}(t-\tau)\right]+\frac{1}{2}\left[\dot{q}_{2}^{2}(t)+\dot{q}_{2}^{2}(t-\tau)\right] .
$$

The nonholonomic constraint is [14]

$$
f=\dot{q}_{1}+b t \dot{q}_{2}-b q_{2}+t=0, \quad(b=\text { const. })
$$

and the boundary conditions are satisfied: when $t \in\left[t_{1}-\tau, t_{1}\right]$, $q_{s}(t)=\Omega_{s}(t)(s=1,2), \Omega_{s}(t)$ is a given piecewise smooth function in $\left[t_{1}-\tau, t_{1}\right]$, where $\tau<t_{2}-t_{1}$ is a given real number, and when $t=t_{2}, q_{s}(t)=q_{s}\left(t_{2}\right)(s=1,2), q_{s}\left(t_{2}\right)$ are certain values.

The differential equations of motion can be expressed as

$$
\begin{aligned}
& \ddot{q}_{1}(t)+\ddot{q}_{1 \tau}(t+\tau)=\lambda=\Lambda_{1}, \\
& \ddot{q}_{2}(t)+\ddot{q}_{2 \tau}(t+\tau)=\lambda b t=\Lambda_{2}, \\
& t_{1} \leq t \leq t_{2}-\tau, \\
& \ddot{q}_{1}(t)=\lambda=\Lambda_{1}, \quad \ddot{q}_{2}(t)=\lambda b t=\Lambda_{2}, \\
& t_{2}-\tau<t \leq t_{2} .
\end{aligned}
$$

Taking derivative of (48) with respect to $t$, we have

$$
\frac{d f}{d t}=\ddot{q}_{1}+b t \ddot{q}_{2}+1=0 .
$$

From formulae (49) and (50), we have

$$
\begin{array}{ll}
\lambda=-\frac{2}{1+b^{2} t^{2}}, & t_{1} \leq t \leq t_{2}-\tau, \\
\lambda=-\frac{1}{1+b^{2} t^{2}}, & t_{2}-\tau<t \leq t_{2} .
\end{array}
$$

Conditions (32) and (33) give

$$
\begin{aligned}
& \left(\dot{q}_{1}(t)+\dot{q}_{1 \tau}(t+\tau)\right)\left(\dot{\xi}_{1}-\dot{q}_{1} \dot{\xi}_{0}\right)+\left(\dot{q}_{2}(t)+\dot{q}_{2 \tau}(t+\tau)\right) \\
& \cdot\left(\dot{\xi}_{2}-\dot{q}_{2} \dot{\xi}_{0}\right)+L \dot{\xi}+\Lambda_{1}\left(\xi_{1}-\dot{q}_{1} \xi_{0}\right) \\
& \quad+\Lambda_{2}\left(\xi_{2}-\dot{q}_{2} \xi_{0}\right)=-\dot{G}, \quad t_{1} \leq t \leq t_{2}-\tau, \\
& \dot{q}_{1}\left(\dot{\xi}_{1}-\dot{q}_{1} \dot{\xi}_{0}\right)+\dot{q}_{2}\left(\dot{\xi}_{2}-\dot{q}_{2} \dot{\xi}_{0}\right)+L \dot{\xi}_{0}+\Lambda_{1}\left(\xi_{1}-\dot{q}_{1} \xi_{0}\right) \\
& \quad+\Lambda_{2}\left(\xi_{2}-\dot{q}_{2} \xi_{0}\right)=-\dot{G}, \quad t_{2}-\tau<t \leq t_{2} .
\end{aligned}
$$

Equations in (52) have the following solutions:

$$
\begin{array}{r}
\xi_{0}^{1}=1, \quad \xi_{1}^{1}=\dot{q}_{1}(t), \quad \xi_{2}^{1}=\dot{q}_{2}(t), \\
G^{1}=-\left(\dot{q}_{1}^{2}(t)+\dot{q}_{2}^{2}(t)\right), \\
t_{1} \leq t \leq t_{2}-\tau, \\
\xi_{0}^{1}=1, \quad \xi_{1}^{1}=\dot{q}_{1}(t), \quad \dot{q}_{2}(t), \\
G^{1}=-\frac{1}{2}\left(\dot{q}_{1}^{2}(t)+\dot{q}_{2}^{2}(t)\right), \\
t_{2}-\tau<t \leq t_{2} ;
\end{array}
$$

$\xi_{0}^{2}=0, \quad \xi_{1}^{2}=1, \quad \xi_{2}^{2}=0$,

$$
G^{2}=\frac{2}{b} \arctan b t
$$$$
t_{1} \leq t \leq t_{2}-\tau,
$$

$\xi_{0}^{2}=0, \quad \xi_{1}^{2}=1, \quad \xi_{2}^{2}=0$,

$$
\begin{array}{r}
G^{2}=\frac{1}{b} \arctan b t, \\
t_{2}-\tau<t \leq t_{2} ;
\end{array}
$$

$$
\xi_{0}^{3}=0, \quad \xi_{1}^{3}=0, \quad \xi_{2}^{3}=1,
$$

$$
G^{2}=\frac{1}{b} \ln \left(1+b^{2} t^{2}\right),
$$$$
t_{1} \leq t \leq t_{2}-\tau,
$$

$\xi_{0}^{3}=0, \quad \xi_{1}^{3}=0, \quad \xi_{2}^{3}=1$,

$$
G^{2}=\frac{1}{2 b} \ln \left(1+b^{2} t^{2}\right),
$$

$$
t_{2}-\tau<t \leq t_{2} ;
$$

$\xi_{0}^{4}=0, \quad \xi_{1}^{4}=-b t, \quad \xi_{2}^{4}=1$,

$$
\begin{array}{r}
G^{4}=b\left(q(t)+q_{\tau}(t+\tau)\right), \\
t_{1} \leq t \leq t_{2}-\tau, \\
\xi_{0}^{4}=0, \quad \xi_{2}^{4}=1, \\
\xi_{1}^{4}=b q(t), \\
t_{2}-\tau<t \leq t_{2} .
\end{array}
$$

These generating functions and gauge functions (53), (54), (55), and (56) correspond to the generalized quasi-symmetrical transformations of the corresponding holonomic system with time delay. The Appell-Chetaev condition (41) gives

$$
\bar{\xi}_{1}+b t \bar{\xi}_{2}=0 .
$$

Formulae (54) and (55) do not satisfy (57), but formulae (53) and (56) satisfy (57). Therefore, formulae (53) and (56) correspond to the generalized quasi-symmetrical transformations 
of nonholonomic system with time delay. Theorem 7 gives the conserved quantities as follows:

$$
\begin{aligned}
I^{1}= & \frac{1}{2}\left[-\dot{q}_{1}^{2}(t)+\dot{q}_{1}^{2}(t-\tau)\right] \\
& +\frac{1}{2}\left[-\dot{q}_{2}^{2}(t)+\dot{q}_{2}^{2}(t-\tau)\right]=\text { const., } \quad t_{1} \leq t \leq t_{2}-\tau, \\
I^{1}= & \frac{1}{2} \dot{q}_{1}^{2}(t-\tau)+\frac{1}{2} \dot{q}_{2}^{2}(t-\tau)=\text { const., } \quad t_{2}-\tau<t \leq t_{2}, \\
I^{4}= & -b t\left(\dot{q}_{1}(t)+\dot{q}_{1 \tau}(t+\tau)\right)+\left(\dot{q}_{2}(t)+\dot{q}_{2 \tau}(t+\tau)\right) \\
& +b\left(q_{1}(t)+q_{1 \tau}(t+\tau)\right)=\text { const., } \quad t_{1} \leq t \leq t_{2}-\tau, \\
I^{4}= & -b t \dot{q}_{1}(t)+\dot{q}_{2}(t)+b q_{1}(t)=\text { const., } \quad t_{2}-\tau<t \leq t_{2} .
\end{aligned}
$$

Formulae in (58) are the conserved quantities corresponding to the Noether generalized quasi-symmetric transformations (53) and (56) for nonholonomic system with time delay that we discussed.

\section{Conclusions}

In this paper, we focus on studying the Noether theorem for nonholonomic systems with time delay. The differential equations for nonholonomic systems with time delay are established. The Noether theorem for nonholonomic systems with time delay is obtained. According to Theorems 6 and 7 , the symmetry problems for nonholonomic systems with time delay can be expressed as the symmetry problems for the corresponding holonomic systems with time delay, which are as the classical nonholonomic system. If $\tau=0$, the nonholonomic systems with time delay can be reduced to the classical nonholonomic systems. The results of this paper are of universal significance, which can be further applied to various types of optimal control systems with time delay, constrained Birkhoffian systems with time delay, and so on.

\section{Conflict of Interests}

The authors declare that there is no conflict of interests regarding the publication of this paper.

\section{Acknowledgment}

This paper is supported by the National Natural Science Foundation of China under Grants nos. 10972151 and 11272227.

\section{References}

[1] H. Y. Hu and Z. H. Wang, "Review on nonlinear dynamic systems involving time delays," Advances in Mechanics, vol. 29, no. 4, pp. 501-512, 1999 (Chinese).

[2] J. Xu and L. J. Pei, "Advances in dynamic for delayed systems," Advances in Mechanics, vol. 36, no. 1, pp. 17-30, 2006 (Chinese).
[3] J. Xu and K. W. Chung, "Dynamics for a class of nonlinear systems with time delay," Chaos, Solitons and Fractals, vol. 40, no. 1, pp. 28-49, 2009.

[4] L. E. El'sgol'c, Qualitative Methods in Mathematical Analysis, American Mathematical Society, Providence, RI, USA, 1964.

[5] D. K. Hughes, "Variational and optimal control problems with delayed argument," Journal of Optimization Theory and Applications, vol. 2, pp. 1-14, 1968.

[6] W. J. Palm and W. E. Schmitendorf, "Conjugate-point conditions for variational problems with delayed argument," Journal of Optimization Theory and Applications, vol. 14, no. 6, pp. 599$612,1974$.

[7] J. F. Rosenblueth, "Systems with time delay in the calculus of variations: a variational approach," IMA Journal of Mathematical Control and Information, vol. 5, no. 2, pp. 125-145, 1988.

[8] W. L. Chan and S. P. Yung, "Sufficient conditions for variational problems with delayed argument," Journal of Optimization Theory and Applications, vol. 76, no. 1, pp. 131-144, 1993.

[9] C. H. Lee and S. P. Yung, "Sufficient conditions for optimal control problems with time delay," Journal of Optimization Theory and Applications, vol. 88, no. 1, pp. 157-176, 1996.

[10] O. P. Agrawal, J. Gregory, and K. Pericak-Spector, "A Bliss-type multiplier rule for constrained variational problems with time delay," Journal of Mathematical Analysis and Applications, vol. 210, no. 2, pp. 702-711, 1997.

[11] G. W. Bluman and S. Kumei, Symmetries and Differential Equations, Springer, New York, NY, USA, 1989.

[12] D. S. Djukic and B. D. Vujanovic, "Noether's theory in classical nonconservative mechanics," Acta Mechanica, vol. 23, no. 1-2, pp. 17-27, 1975.

[13] M. Lutzky, "Dynamical symmetries and conserved quantities," Journal of Physics A: Mathematical and General, vol. 12, no. 7, pp. 973-981, 1979.

[14] F. X. Mei, Applications of Lie Groups and Algebras to Constrained Mechanical Systems, Science Press, Beijing, China, 1999 (Chinese).

[15] F. X. Mei, "Lie symmetries and conserved quantities of constrained mechanical systems," Acta Mechanica, vol. 141, no. 3-4, pp. 135-148, 2000.

[16] F. X. Mei, "Advances in the symmetries and conserved quantities of classical constrained systems," Advances in Mechanics, vol. 39, no. 1, pp. 37-43, 2009 (Chinese).

[17] G. S. F. Frederico and D. F. Torres, "A formulation of Noether's theorem for fractional problems of the calculus of variations," Journal of Mathematical Analysis and Applications, vol. 334, no. 2, pp. 834-846, 2007.

[18] T. M. Atanacković, S. Konjik, S. Pilipović, and S. Simić, "Variational problems with fractional derivatives: invariance conditions and Nöther's theorem," Nonlinear Analysis: Theory, Methods \& Applications, vol. 71, no. 5-6, pp. 1504-1517, 2009.

[19] S. K. Luo, Z. J. Li, W. Peng, and L. Li, "A lie symmetrical basic integral variable relation and a new conservation law for generalized Hamiltonian systems," Acta Mechanica, vol. 224, no. 1, pp. 71-84, 2013.

[20] Y. Zhang and Y. Zhou, "Symmetries and conserved quantities for fractional action-like Pfaffian variational problems," Nonlinear Dynamics, vol. 73, no. 1-2, pp. 783-793, 2013.

[21] Y. Zhang and X.-H. Zhai, "Noether symmetries and conserved quantities for fractional Birkhoffian systems," Nonlinear Dynamics, 2015. 
[22] V. I. Arnold, V. V. Kozlov, and A. I. Neishtadt, Mathematical Aspects of Classical and Celestial Mechanics, Springer, Berlin, Germany, 2006.

[23] D. I. Bower, An Introduction to Polymer Physics, Cambridge University Press, Cambridge, UK, 2002.

[24] G. S. Frederico and D. F. Torres, "Noether's symmetry theorem for variational and optimal control problems with time delay," Numerical Algebra, Control and Optimization, vol. 2, no. 3, pp. 619-630, 2012.

[25] Y. Zhang and S. X. Jin, "The Noether symmetries of dynamics for non-holonomic systems with time delay," Acta Physica Sinica, vol. 62, no. 23, Article ID 234502, 9 pages, 2013 (Chinese).

[26] S.-X. Jin and Y. Zhang, "Noether symmetry and conserved quantity for a Hamilton system with time delay," Chinese Physics $B$, vol. 23, no. 5, Article ID 054501, 8 pages, 2014.

[27] X.-H. Zhai and Y. Zhang, "Noether symmetries and conserved quantities for Birkhoffian systems with time delay," Nonlinear Dynamics, vol. 77, no. 1-2, pp. 73-86, 2014.

[28] F. X. Mei, "Nonholonomic mechanics," Applied Mechanics Reviews, vol. 53, no. 11, pp. 283-305, 2000.

[29] J. I. Neimark and N. A. Fufaev, Dynamics of Nonholonmic Systems, AMS, Providence, RI, USA, 1972.

[30] V. V. Kozlov, "The dynamics of systems with nonintegrable constraints I," Vestnik Moskovskogo Universiteta. Seriya I. Matematika, Mekhanika, vol. 1, no. 3, pp. 92-100, 1982.

[31] F. X. Mei, Analytical Mechanics II, Beijing Institute of Technology Press, Beijing, China, 2013.

[32] J. G. Papastavridis, "A panoramic overview of the principles and equations of motion of advanced engineering dynamics," Applied Mechanics Reviews, vol. 51, no. 4, pp. 239-265, 1998. 


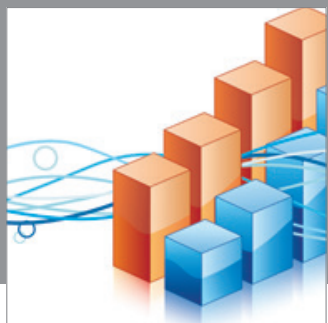

Advances in

Operations Research

mansans

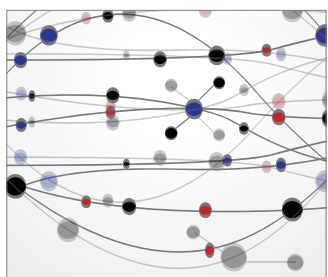

The Scientific World Journal
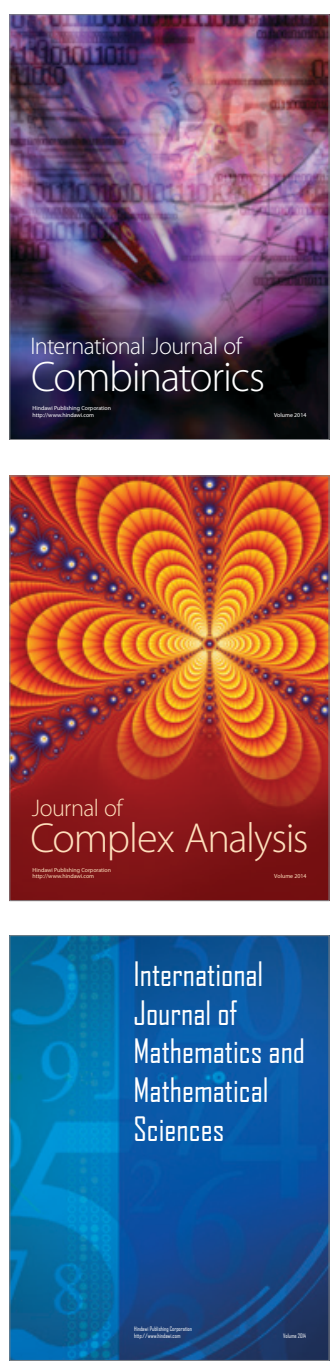
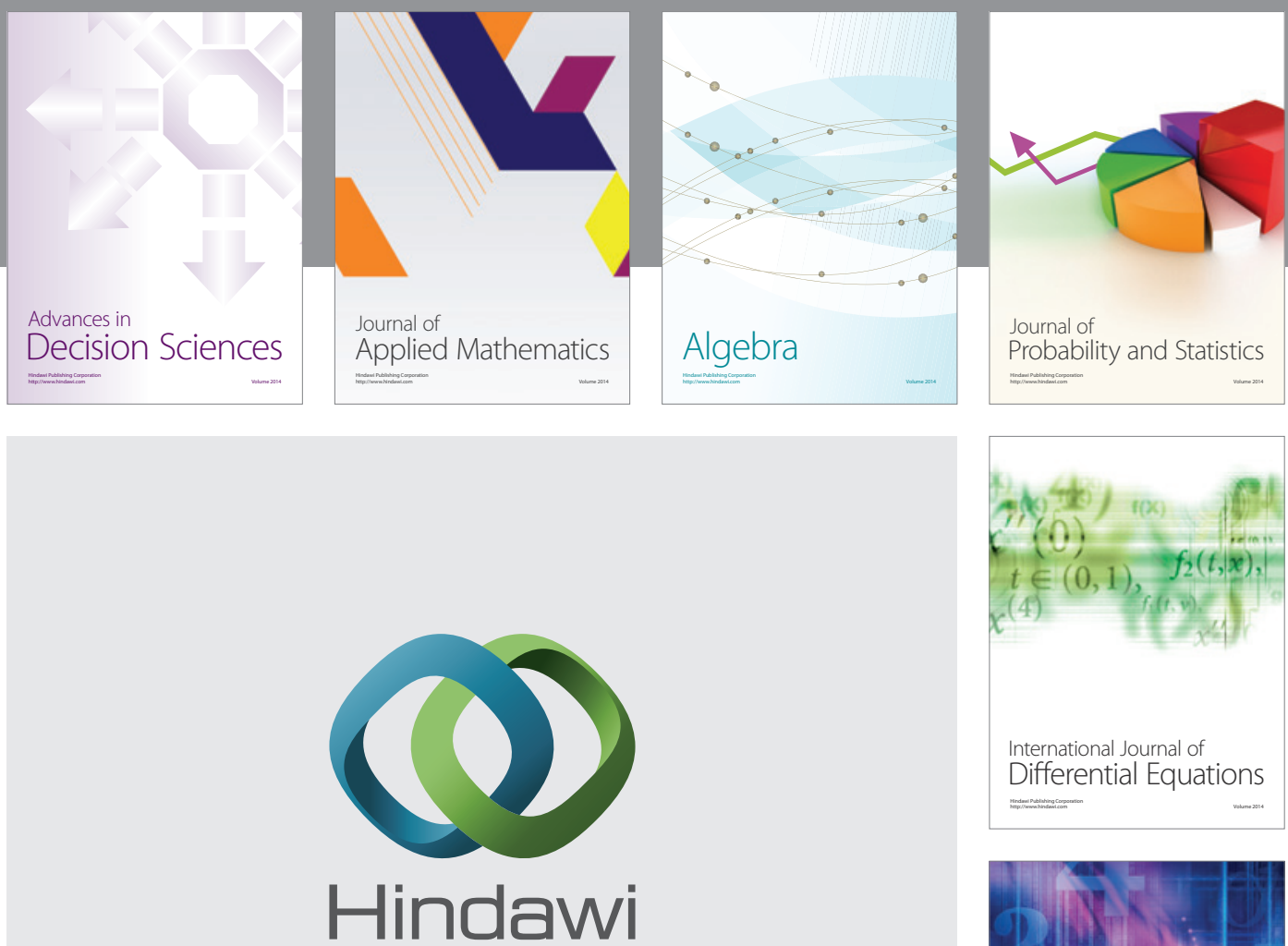

Submit your manuscripts at http://www.hindawi.com
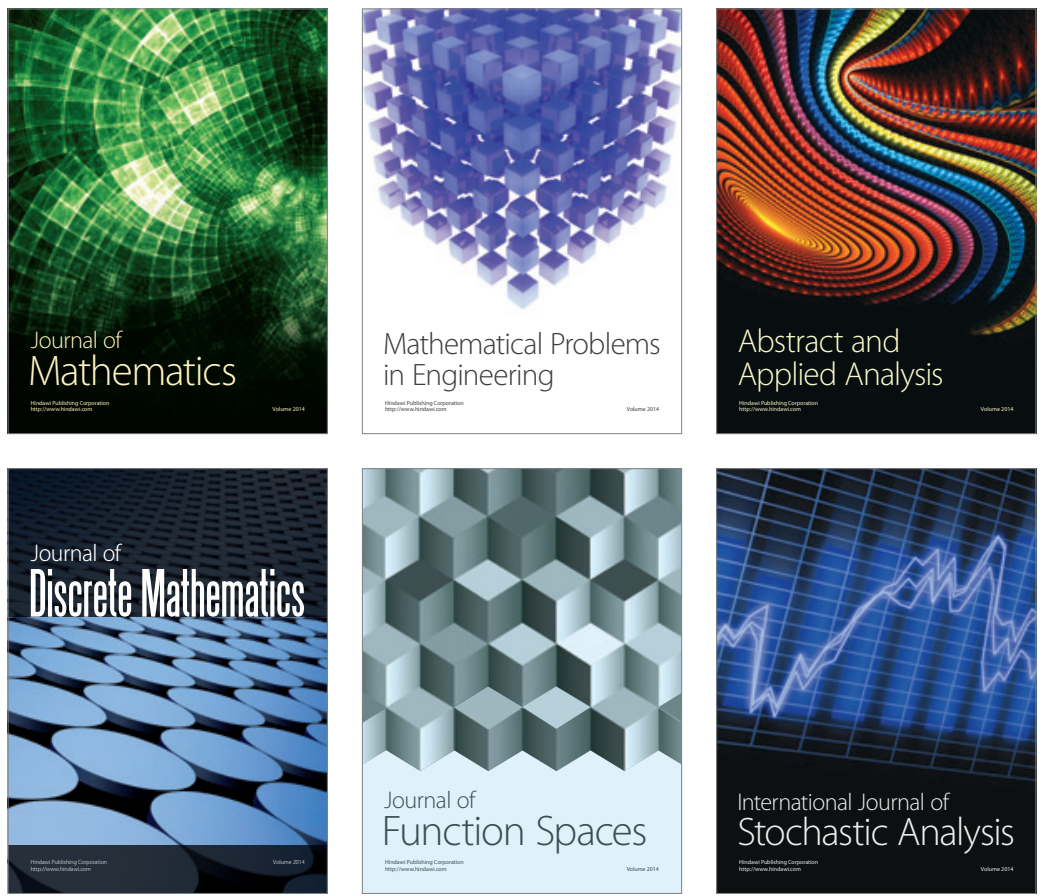

Journal of

Function Spaces

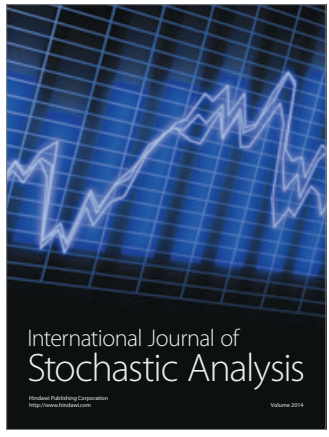

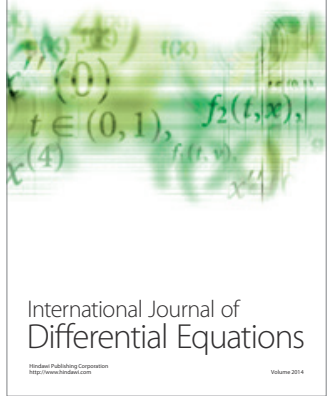
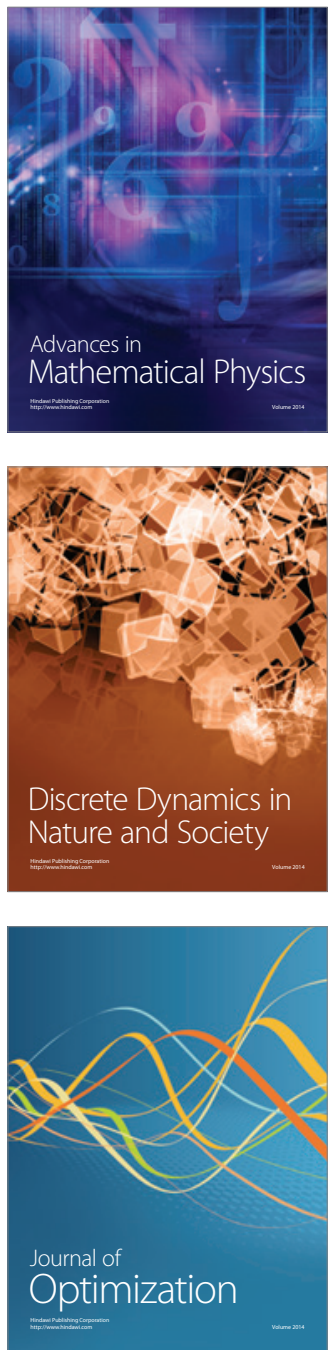